\title{
Porcine aortic surgical model to study isolated intraspinal collateralization
}

\author{
Fabian A. Kari, MD, Matthias Siepe, MD, and Friedhelm Beyersdorf, MD, Freiburg, Germany
}

See related commentary on pages 415-6.

The function of the intraspinal collateral system, which includes repetitive epidural arcades and anterior radiculomedullary arteries, is not well understood in the setting of segmental artery ligation during aortic surgery. The animal model presented here can be used to characterize the function of the intraspinal collateral backup system in detail.

\section{BACKGROUND}

The incidence of spinal cord ischemia during extensive aortic surgery is still reported to be as high as $15 \%$ to $20 \%$. Preoperative risk prediction according to individual collateral anatomy is not currently possible. The porcine model has been established as a valuable opportunity to study spinal collateral physiology in an aortic surgical setting. ${ }^{1}$ The intraspinal compartment of the spinal collateral network includes a serial pattern of circular arcades and anterior radiculomedullary arteries (ARMAs) ${ }^{1}$ and has not yet been functionally characterized. We postulate an important contribution of this system to immediate recovery of a sufficient spinal cord perfusion pressure directly after serial occlusion of segmental vessels. We sought to create a large animal model with spinal collateral flow being exclusively delivered through the intraspinal system. In a proof-of-concept study, a shutdown of thoracic and lumbar paraspinous inflow was achieved through percutaneous ethanol ablation of dorsal segmental arteries. The goal was to create a model suitable for investigation of the intraspinal collateral system in isolation, to understand more clearly its role in maintaining adequate spinal cord perfusion during aortic surgery.

\section{ANIMAL MODEL AND METHODS}

Six juvenile pigs (mean weight of $25 \mathrm{~kg}$ ) underwent bilateral percutaneous ethanol ablation of the paraspinous aspect of the dorsal lumbar and thoracic segmental arteries (T3-L5) while under general anesthesia.

\footnotetext{
From the Department of Cardiovascular Surgery, Heart Center, Freiburg University, Freiburg, Germany.

Disclosures: Authors have nothing to disclose with regard to commercial support.

Received for publication Jan 22, 2015; revisions received March 1, 2015; accepted for publication March 3, 2015; available ahead of print April 1, 2015.

Address for reprints: Fabian A. Kari, MD, Heart Center, Freiburg University, Hugstetter Strasse 55, 79104 Freiburg, Germany (E-mail: Fabian.alexander.kari@ universitaets-herzzentrum.de).

J Thorac Cardiovasc Surg 2015;150:413-4

$0022-5223 / \$ 36.00$

Copyright (c) 2015 by The American Association for Thoracic Surgery

http://dx.doi.org/10.1016/j.jtcvs.2015.03.001
}

The anatomic landmark for injections was the cranial margin of the respective vertebral lamina. Preinterventional patency of these vessels was confirmed by thin-slice computed tomographic angiography and magnetic resonance angiography. The animals were followed up for 5 days, and the modified Tarlov score was documented (score ranging from 0 to 9 , with 0 for flaccid paraplegia, 1 for weak motor reaction on sensory stimulus, 2 for strong motor reaction on sensory stimulus and ability to stand, 3 for assisted standing $<1$ minute, 4 for assisted standing up and standing without assistance $<1$ minute, 5 for standing up and standing without assistance $>1$ minute, 7 for walking $<1$ minute, 8 for walking $>1$ minute, and 9 for complete recovery). Magnetic resonance and computed tomographic angiographic controls were done postinterventionally to verify successful shutdown of paraspinous inflow and muscular tissue reaction to percutaneous ethanol ablation ( $5 \mathrm{~mL}$ ethanol per segmental level and side). After the animals were killed, paraspinous necrosis and scarring, as well as the absence of patent paraspinous vessels, were verified both macroscopically and microscopically. The local institutional review board approved the reported experiments.

\section{PRELIMINARY RESULTS}

Successful shutdown of paraspinous inflow into the spinal collateral network was confirmed in postinterventional controls (Figure 1). Postinterventional magnetic resonance imaging of the surrounding connective and muscular tissue showed reactive edema. After the animals were killed, limited (mean size $10 \pm 3 \mathrm{~mm}$ ) necrotic lesions in the paraspinous muscular tissue were found to be present at each level and bilaterally. All but 1 animal regained normal motor function of the hind limbs (Figure 2). Postoperative analgesia and slight temperature increase could be managed with nonsteroidal analgesics alone. The animals were lively and unchanged with regard to their behavior before the intervention. One animal remained paraparetic throughout the follow-up period, probably because of the effect on the segmental nerves of the percutaneous ethanol ablation (Figure 2). This animal did not regain the ability to stand without assistance, although there were weak motor reactions on sensory stimulus. After 3 days of follow-up during which the Tarlov scores were less than 2, this animal was killed according to the experimental protocol. Another animal had weakness develop in the paraspinous musculature, manifesting as a slight increase of concavity of the lumbar spine; however, it did not demonstrate any deficits in motor function. Standard histologic staining revealed massive necrosis and macrophage infiltration with complete obliteration of the major paraspinous vasculature.

\section{DISCUSSION}

The collateral network of the spinal cord surrounds the dorsal parts of the bony vertebral column. It is supplied 


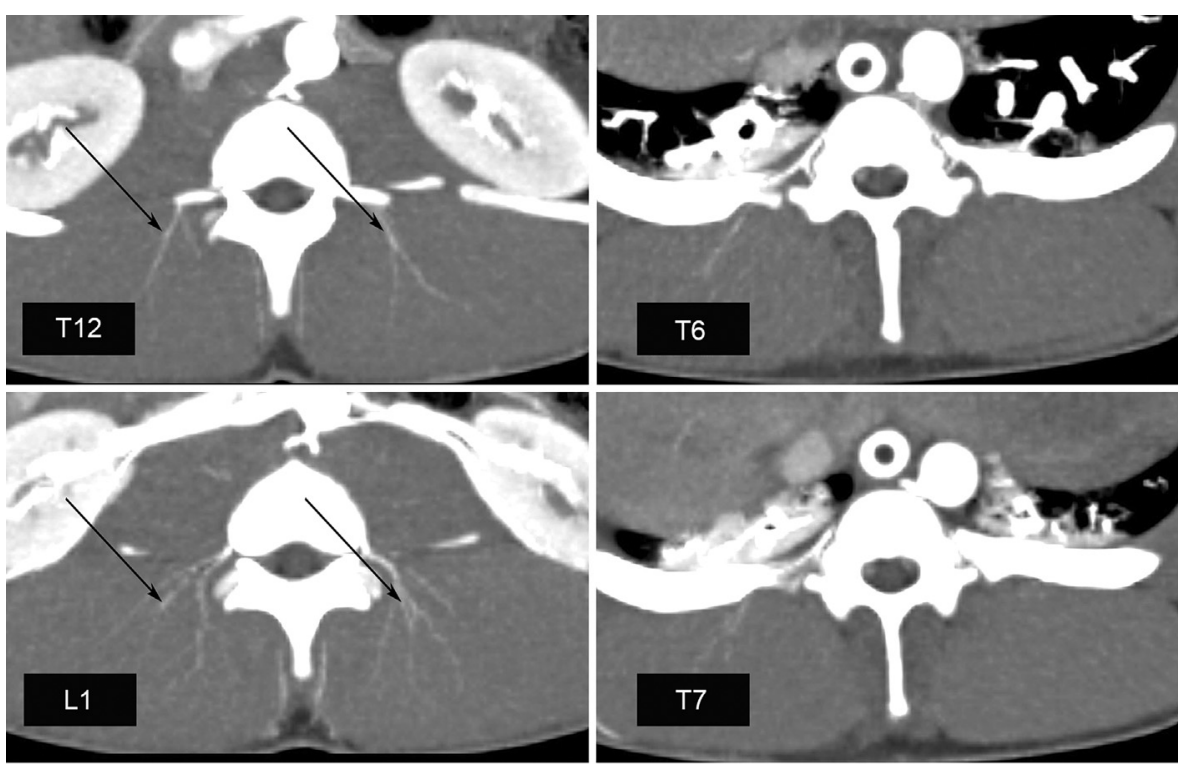

FIGURE 1. Untreated (left) and treated (right) segments after ablation of dorsal paraspinous arterial branches. Patent paraspinous arterial trees marked by black arrows can be seen in untreated segments; no patent paraspinous vessels are visible in treated segments.

by different main sources: cranial branches arise from the supra-aortic vessels, and caudal branches arise from the hypogastric arteries, the iliac arteries, and the median sacral artery. ${ }^{2}$ The network is furthermore segmentally augmented by dorsal branches of the segmental arteries, specifically the intercostal and lumbar arteries.

The spinal collateral network ${ }^{2}$ can be divided into paraspinous and intraspinal compartments. In the paraspinous compartment, small natively nonconducting arteriolar vessels can be found, which likely undergo arteriogenesis ${ }^{3}$ midterm to long term after ligation of main segmental arteries during aortic surgery. ${ }^{1}$ In the intraspinal system, a repetitive pattern of circular or pentagonal small conducting arteries is found, which connects

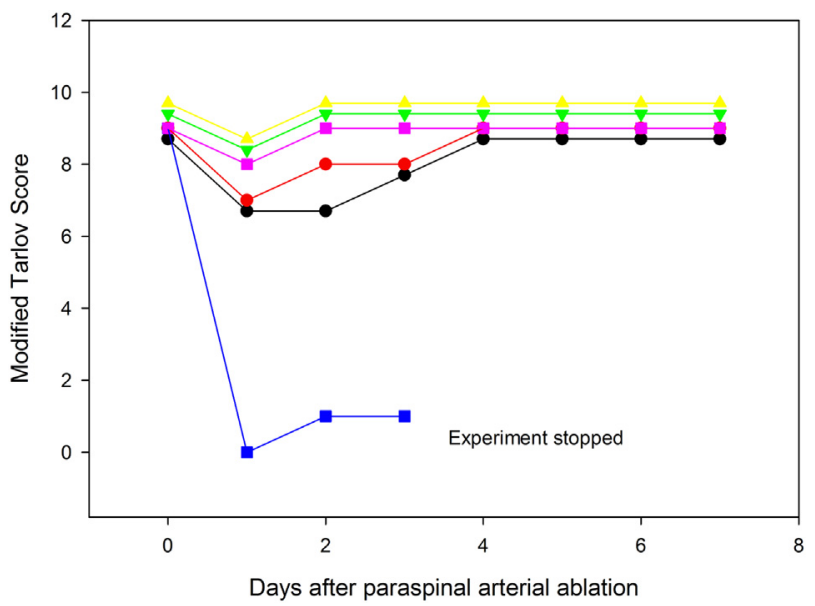

FIGURE 2. Modified Tarlov scores after paraspinous artery ablation. In the case of the single animal in which Tarlov score dropped to 0 and did not recover even to a score of 2 , the experiment was terminated on day 3 , and the animal was sacrificed at that time. neighboring segments longitudinally and side-to-side. The ARMAs connecting the dorsal segmental arteries with the anterior spinal artery are the major variable part of this collateral system. The number (from 3 to 15 in human beings) and distribution along the length of the spine, as well as sidedness, are highly variable among individuals. ${ }^{4}$ To date, no solid risk prediction of spinal ischemia is possible preoperatively for patients undergoing thoracic aortic surgery according to individual number and distribution of ARMAs.

As imaging modalities for spinal arteries, including ARMAs, continue to progress, ${ }^{5}$ a better understanding of the intraspinal immediate backup collateral system is necessary. The model presented here can be a way to characterize the importance and physiologic details of the intraspinal backup system by shutting down inflow from paraspinous collaterals. Understanding the exact function of the intraspinal collaterals, including ARMA number and distribution, can help improving preoperative risk assessment for aortic surgical patients.

\section{References}

1. Etz CD, Kari FA, Mueller CS, Silovitz D, Brenner RM, Lin HM, et al. The collateral network concept: a reassessment of the anatomy of spinal cord perfusion. $J$ Thorac Cardiovasc Surg. 2011;141:1020-8.

2. Griepp RB, Ergin MA, Galla JD, Lansman S, Khan N, Quintana C, et al. Looking for the artery of Adamkiewicz: a quest to minimize paraplegia after operations for aneurysms of the descending thoracic and thoracoabdominal aorta. J Thorac Cardiovasc Surg. 1996;112:1202-13; discussion 1213-5.

3. Buschmann I, Schaper W. Arteriogenesis versus angiogenesis: two mechanisms of vessel growth. News Physiol Sci. 1999;14:121-5.

4. Lazorthes G, Poulhes J, Bastide G, Roulleau J, Chancholle AR. [Arterial vascularization of the spine; anatomic research and applications in pathology of the spinal cord and aorta]. Neurochirurgie. 1958;4:3-19. French.

5. Nijenhuis RJ, Backes WH. Optimal preoperative imaging of spinal cord blood supply. AJNR Am J Neuroradiol. 2009;30:E38-9. author reply E40. 